\title{
Agencia de autocuidado en la vida diaria del adulto mayor que vive con diabetes mellitus tipo 2 antes y después del apoyo educativo de enfermería
}

\section{Self-care Agency in the Daily Life of the Elderly Person Living with Type 2 Diabetes Mellitus Before and After Nursing Education Support}

\author{
Fabiola I. Peza-Alejandre ${ }^{a}$, Gabriela Maldonado-Muñiz ${ }^{b}$,Claudia A. Trejo-García ${ }^{c}$
}

\begin{abstract}
:
Introduction: the elderly have a high prevalence of chronic-degenerative diseases in Mexico, the prevalence of diabetes mellitus has been growing rapidly, especially in the elderly. Objective: To evaluate the self-care agency in the daily life of the elderly person living with diabetes mellitus 2, before and after nursing education support. Methods and materials: quantitative study, of correlational, quasiexperimental scope, included elderly people living with diabetes mellitus 2, who attended the Centro Gerotológico de Tula. The Scale of Appreciation of Self-Care Agency (ASA) capacity was applied, structuring before and after the educational support directed to the elderly living with diabetes mellitus 2 to improve the self-care agency. Descriptive and inferential statistics were used: Wilcoxon $(\mathrm{p} \leq 0.05$ ). Results: $91.7 \%$ female gender; $8.3 \%$ were single, $50 \%$ married and $41.7 \%$ widowed. The mean age was $71.08 \pm 5.3$ years. In the evaluation of the self-care agency, $83.3 \%$ were found in the first measurement with a very good rating and 16.7 with a good rating, and a very good $100 \%$ in the second measurement. Wilcoxon $z=-2.572 \mathrm{p}=.01$. Conclusions: The self-care agency in older adults living with type 2 diabetes mellitus increases after educational support.
\end{abstract}

Keywords:

Elderly, Self-care agency, Diabetes Mellitus Type 2, Nursing Education Support.

\section{Resumen:}

Introducción: los adultos mayores presentan una alta prevalencia de enfermedades crónico-degenerativas en México, la prevalencia diabetes mellitus ha ido creciendo de manera acelerada, especialmente en el adulto mayor. Objetivo: Evaluar agencia de autocuidado en la vida diaria del adulto mayor que vive con diabetes mellitus 2, antes y después del apoyo educativo de enfermería. Métodos y materiales: estudio cuantitativo, de alcance correlacional, cuasiexperimental, se incluyó a los adultos mayores que viven con diabetes mellitus 2, que asistían al Centro Gerontológico de Tula. Se aplicó la Escala de apreciación de la capacidad de agencia de autocuidado (ASA) estructurando antes y después del apoyo educativo dirigido al adulo mayor que viven con diabetes mellitus 2 para mejorar la agencia de autocuidado. Se utilizó estadística descriptiva e inferencial: Wilcoxon ( $\mathrm{p} \leq 0.05$ ). Resultados: 91.7\% género femenino; 8.3\% eran solteros, $50 \%$ casados y $41.7 \%$ viudos. La media de edad fue $71.08 \pm 5.3$ años. En la evaluación de agencia de autocuidado se encontró en la primera medición $83.3 \%$ con calificación muy buena y 16.7 buena, pasando al 100\% muy buena en la segunda medición. Wilcoxon $\mathrm{z}=-2.572 \mathrm{p}=.01$. Conclusiones: La agencia de autocuidado en los adultos mayores que viven con diabetes mellitus tipo 2 aumenta posterior al apoyo educativo.

\section{Palabras Clave:}

Adulto Mayor, Agencia de Autocuidado, Diabetes Mellitus Tipo 2, Apoyo Educativo de Enfermería.

\footnotetext{
a Alumna de la Universidad Autónoma del Estado de Hidalgo, Escuela Superior de Tlahuelilpan. E-mail: fabiolaalejandre@ hotmail.com

${ }^{\text {b }}$ Profesor Investigador de la Universidad Autónoma del Estado de Hidalgo, Escuela Superior de Tlahuelilpan. E-mail:

gmaldonado@uaeh.edu.mx

c Autor de Correspondencia, Profesor Investigador de la Universidad Autónoma del Estado de Hidalgo, Escuela Superior de Tlahuelilpan. 


\section{Introducción}

La Organización Mundial de la Salud (OMS), considera adultos mayores a las personas de más de 60 años para los que viven en los países en vías de desarrollo y de 65 años a los que viven en países desarrollados. (Instituto para la Atención de los Adultos Mayores en la ciudad de México, s.f.)

El envejecimiento se puede definir como un fenómeno presente a lo largo del ciclo de la vida desde la concepción hasta la muerte

La diabetes mellitus es uno de los principales problemas de salud pública que afectan a los adultos mayores en estos últimos años, ha ido creciendo de manera acelerada en México. Si se diagnóstica en estado avanzado y se le da un tratamiento inadecuado, se pueden ocasionar serias complicaciones de salud, así como la muerte prematura. (Salcedo-Álvarez et al., 2017) A escala mundial se calcula que 422 millones de adultos tenían diabetes en 2014, por comparación con 108 millones en 1980, esto corresponde con un aumento de sus factores de riesgo, tales como el sobrepeso y la obesidad. (OMS, 2016)

Un elemento vital para la prevención y el tratamiento es el autocuidado, propuesto por la teórica Dorothea Orem, que lo define como las acciones que realiza el propio individuo para mantener la salud y satisfacer sus necesidades vitales.

La presente investigación tuvo como objetivo evaluar la agencia de autocuidado en la vida diaria del adulto mayor que vive con diabetes mellitus tipo 2 antes y después del apoyo educativo de enfermería con la finalidad de comprobar que el apoyo educativo de enfermería aumenta la agencia de autocuidado.

\section{Planteamiento del problema o exposición del caso}

Se estima que en 2015 la diabetes fue la causa directa de 1,6 millones de muertes, (OMS) la incidencia y prevalencia de diabetes mellitus ha alcanzado proporciones muy grandes en todo el mundo. Las razones para esta epidemia están directamente asociadas al aumento de pacientes con obesidad, al sedentarismo, y a la mala alimentación. (Fernández-Vázquez et al., 2012) Estas acciones atraen consecuencias catastróficas como: riesgo 2 a 3 veces mayor de infarto de miocardio, accidente cerebrovascular, riesgo de úlceras de los pies, infección y, en última instancia, amputación. (OMS, 2018)

El apoyo educativo para las personas que viven con diabetes es una herramienta esencial para optimizar el control metabólico y prevenir la aparición y progresión de las complicaciones agudas y crónicas de la enfermedad, y sobre todo en los pacientes adultos mayores. La OMS considera que la educación sanitaria es una parte fundamental en el tratamiento de la diabetes y la única eficaz para el control de la enfermedad y la prevención de sus complicaciones. (González Pedraza-Avilés, et al, 2007)

He aquí la importancia de la participación del profesional de enfermería en la salud comunitaria hacia la prevención primaria; haciendo énfasis en enseñar, educar, motivar responsabilidades de acuerdo con sus necesidades y capacidades funcionales en cada uno de los adultos mayores.

Por lo que se plantea la siguiente pregunta: ¿Cuál es la Agencia de Autocuidado en la Vida Diaria del Adulto Mayor que Vive con Diabetes Mellitus tipo II, Antes y Después del Apoyo Educativo de Enfermería?

\section{Conocimiento previo}

Entre el 2015 y el 2050 la proporción de la población mundial con más de 60 años de edad pasara a 900 millones hasta 2000 millones lo que representa un amiento del $12 \%$ al $22 \%$. (OMS, 2015)

Según la encuesta nacional de salud y nutrición 2012, la diabetes se encuentra entre las primeras causas de muerte en México. 4 millones de personas refirieron haber sido diagnosticadas con diabetes.

El incremento en actividad física, dieta adecuada y reducción de peso, disminuyen el riesgo de desarrollar diabetes entre $34 \%$ y $43 \%$, efecto que puede durar de 10 a 20 años después de concluida la intervención. (Federación Mexicana de Diabetes, 2014)

En la revisión bibliográfica se encontraron diversos artículos de interés que nutren la investigación, como: el estudio de Loredo Figueroa y colaboradores, con el título de Nivel de dependencia, autocuidado y calidad de vida del adulto mayor realizado en una unidad de medicina familiar del Instituto de Seguridad y Servicios Sociales de los Trabajadores del Estado (ISSSTE) en Querétaro en el año 2016, se encontró que los adultos mayores tienen un autocuidado consiente, el hecho de que sean personas que aún mantienen cierta independencia de acuerdo con su edad, se sienten capaces de cumplir con su autocuidado y lo realizan de una manera efectiva gracias a la información que se les proporciona en su unidad médica. (Loredo-Figueroa, et al, 2016).

En un estudio realizado en la Unidad de Atención Básica de Coomeva en Cartagena, Colombia, realizado por Herrera Lían y colaboradores, con 157 pacientes diagnosticados con diabetes mellitus tipo 2, encontraron que el $62,4 \%$ de los diabéticos tenían un control metabólico inadecuado, debido al mal seguimiento del 
tratamiento. Reportan que, dentro de la población estudiada, las mujeres fueron las que tuvieron el índice más alto en la deficiencia de autocuidado, y de igual manera las personas con recursos económicos bajos (Herrera-Lían, et al, 2012).

\section{Metodología de estudio}

Tipo de estudio aplicado, medición de las variables cuantitativa con alcance correlacional. Diseño metodológico experimental, cuasiexperimental, prolectivo, longitudinal, prospectivo, con universo de 12 adultos mayores que viven con diabetes mellitus 2 . El estudio se realizó en el centro gerontológico de Tula de Allende, Hidalgo, el trabajo de campo se realizó de junio a julio del 2018. La muestra fue conformada por el universo, se incluyó personas de 65 a 80 años que vivan con diabetes mellitus 2, que estuvieron de acuerdo con participar en la investigación y firmaron el consentimiento informado.

Se aplicó la escala de apreciación de la capacidad de agencia de autocuidado (ASA), se compone de 24 ítems con un formato de respuesta de cuatro alternativas tipo Likert, donde el 1 (totalmente en desacuerdo) significa el valor más bajo de capacidad de autocuidado y el 4 (totalmente de acuerdo) el más alto, con una Confiabilidad y validez de Chi 2 de 563,9 y Alfa de Cronbach 0,84 (84\%).

El apoyo educativo se implementó mediante el programa titulado: "aprendiendo a cuidarme", que se impartió por una hora, dos días a la semana, durante el mes de julio 2018, se proporcionó información sobre cómo vivir con la diabetes mellitus 2 y cuáles pueden ser las estrategias a seguir para lograr una agencia de autocuidado eficaz por parte de los adultos mayores.

Para el análisis de los datos se utilizó estadística descriptiva e inferencial: la prueba de los rangos de Wilcoxon en conjunto con el programa de Excel de Microsoft y SPSS. Para la prueba de hipótesis se consideró estadísticamente significativa si $p \leq 0.05$.

\section{V.Aplicación}

El presente estudio tiene una aplicación en las ciencias de la salud en el área de enfermería con la agencia de autocuidado según el modelo de Dorothea Orem. Ver Figura 1.

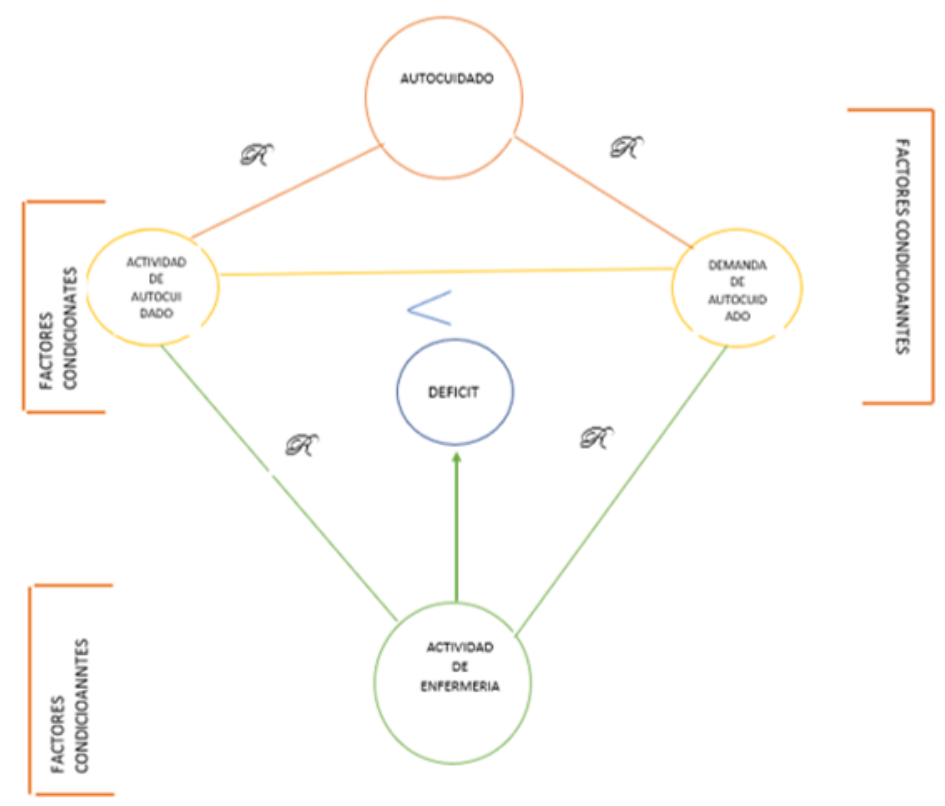

Figura 1. Déficit de autocuidado de D.Orem.

\section{Resultados}

La muestra estuvo conformada por 12 adultos mayores que se categorizaron según grupos de edad, sexo, se evaluaron los conocimientos antes y después del apoyo educativo con los siguientes resultados.

El $91.7 \%(n=11)$ fueron participantes del género femenino, y el $8.3 \%(n=1)$ fueron del género masculino. La edad promedio de los participantes fue de $71.08 \pm 5.3$ años. En la evaluación de agencia de autocuidado se encontró en la primera medición $83.3 \%(n=10)$ una calificación muy buena, y $16.7(n=2)$ buena, pasando al 100\% en $(n=12)$ en muy buena en la segunda medición. La calificación de la capacidad de agencia de autocuidado en la primera medición fue de: $87.42 \pm 4.6$ en la segunda medición, con prueba de rangos Wilcoxon $z=-2.572 p=.01$, lo que es estadísticamente significativo por lo que se aprueba la hipótesis de trabajo que dice: la Agencia de Autocuidado de los Adultos Mayores que viven con Diabetes aumenta con el Apoyo Educativo de enfermería

\section{Discusión}

La agencia de autocuidado de los adultos mayores mejora con el apoyo educativo obteniendo un $83.3 \%(n=10)$ de los adultos mayores que viven con diabetes mellitus donde obtuvieron en la primera medición una calificación muy buena, y $16.7 \%(n=2)$ buena, pasando al $100 \%(n=12)$ en muy buena en la segunda medición, lo que es similar a lo reportado por Ochoa Ortega y cols., en el estudio titulado Estrategia de intervención educativa en adultos mayores diabéticos tipo 2, realizada en el 2012 en Pinar del río, Cuba donde encontraros un nivel de conocimientos bueno 
en la primera medición con $90.20 \%$ pasando al $100 \%$ en la segunda medición, ninguno de los pacientes en los que se realizó la intervención mostro resultados malos. (Ochoa-Ortega, et al., 2012).

Por lo cual podemos destacar que el apoyo educativo de enfermería en adultos mayores que viven con diabetes mellitus ha dado resultados positivos, que aumentan las capacidades de autocuidado y los conocimientos sobre la importancia de llevar un tratamiento adecuado y los beneficios que atrae, en México y en otros países.

\section{Conclusiones}

La agencia de autocuidado en los adultos mayores que viven con diabetes mellitus tipo 2 aumenta posterior al apoyo educativo.

Si bien, los resultados muestran que los adultos mayores mantienen una agencia de autocuidado muy buena, no obstante es necesario continuar con el apoyo educativo fomentando los conocimientos, habilidades y valores que favorezcan el autocuidado para asegurar la salud y protección de los adultos mayores.

\section{Agradecimientos}

A los adultos mayores participantes y a las autoridades del Centro Gerontológico de Tula de Allende, por su apoyo.

\section{Referencias}

Federación Mexicana de Diabetes. (2014). Diabetes en México. Recuperado el 27 de abril de 2019, de Federación Mexicana de Diabetes: http://fmdiabetes.org/diabetes-en-mexico/

Fernández-Vázquez, A., Abdala-Cervantes, T., AlvaraSolís, E., Tenorio-Franco, G., López-Valencia, E., \& Cruz-Centeno, S., Dávila-Mendoza, R., \& González Pedraza-Avilés, A. (2012). Estrategias de autocuidado en pacientes con diabetes mellitus tipo 2. Revista de Especialidades Médico-Quirúrgicas, 17 (2), 94-99. Recuperado en 27 de abril de 2019, de http://www.redalyc.org/articulo.oa?id=47323278006

González Pedraza-Avilés, A., \& Martínez Vázquez, R. (2007). Efectividad de una estrategia educativa sobre los parámetros bioquímicos y el nivel de conocimientos en pacientes diabéticos tipo 2. Revista de Endocrinología y Nutrición, 15(3), 165174. Recuperado el 27 de abril de 2019: http://www.medigraphic.com/pdfs/endoc/er2007/er073g.pdf
Herrera-Lían, A., Andrade, Y., Hernandez, O., Manrrique, J., Faria, K., \& Machado, M. (2012). Personas con diabetes mellitus tipo 2 y su capacidad de agencia de autocuidado, Cartagena. Avances en Enfermería, 30(2), 39-46. Recuperado el 27 de abril de 2019: https://revistas.unal.edu.co/index.php/avenferm/artic le/view/36079

Instituto para la Atención de los Adultos Mayores en la ciudad de México. (s.f.). ¿Quién es la persona mayor? Recuperado el 27 de abril de 2019: http://www.adultomayor.cdmx.gob.mx/index.php/qui en-es-el-adulto-mayor

Loredo-Figueroa, M., Gallegos-Torres, R., XequeMorales, A., Palome-Veja, G., \& Juarez-Lira, A. (2016). Nivel de dependencia, autocuidado y calidad de vida del adulto mayor. Enfermería Universitaria, 13(3), 159-165. Recuperado el 27 de abril de 2019: http://www.scielo.org.mx/scielo.php?pid=S166570632016000300159\&script=sci_arttext\&tlng=en

Ochoa-Ortega, M.R., Díaz-Domínguez, M.Á., CasanovaMoreno, M.C., Pérez-Sierra, M., \& Llogas-Porras, M. (2012). A strategy of an educational intervention in Type-2 diabetic old people. Revista de Ciencias Médicas de Pinar del Río, 16(3), 109-119. Recuperado en 27 de abril de 2019, de http://scielo.sld.cu/scielo.php?script=sci_arttext\&pid $=$ S1561-31942012000300011\&lng=es\&tIng=en.

OMS. (2015). Envejecimiento y salud. Recuperado de Organización Mundial de la Salud, el 27 de abril de 2019: $\quad$ https://www.who.int/es/news-room/factsheets/detail/envejecimiento-y-salud

OMS. (2016). Informe mundial sobre la diabetes. Who, 184. Recuperado de Organización Mundial de la Salud, el 27 de abril de 2019: https://doi.org/10.18004/rvspmi/23123893/2016.03(02)71-076

OMS. (2018). Diabetes. Recuperado de Organización Mundial de la Salud, el 27 de abril de 2019: https://www.who.int/es/news-room/factsheets/detail/diabetes

Salcedo-Álvarez, R.A., Jiménez-Mendoza, A., GonzálezCaamaño, B.C., Rivas-Herrera, J.C., \& del PradoVázquez, A. (2017). Plan domiciliario de autocuidado para adultos mayores con DM2 aplicando la taxonomía NANDANOC-NIC. Rev Enferm Inst Mex Seguro Soc, 25(4), 299-309. Recuperado en 27 de abril de 2019, de https://www.medigraphic.com/pdfs/enfermeriaimss/ eim-2017/eim174i.pdf 